University of Texas at El Paso

ScholarWorks@UTEP

$10-2019$

\title{
Why a Classification Based on Linear Approximation to Dynamical Systems Often Works Well in Nonlinear Cases
}

Julio Urenda

The University of Texas at El Paso, jcurenda@utep.edu

Vladik Kreinovich

The University of Texas at El Paso, vladik@utep.edu

Follow this and additional works at: https://scholarworks.utep.edu/cs_techrep

Part of the Computer Sciences Commons, and the Mathematics Commons Comments:

Technical Report: UTEP-CS-19-103

\section{Recommended Citation}

Urenda, Julio and Kreinovich, Vladik, "Why a Classification Based on Linear Approximation to Dynamical Systems Often Works Well in Nonlinear Cases" (2019). Departmental Technical Reports (CS). 1377. https://scholarworks.utep.edu/cs_techrep/1377

This Article is brought to you for free and open access by the Computer Science at ScholarWorks@UTEP. It has been accepted for inclusion in Departmental Technical Reports (CS) by an authorized administrator of ScholarWorks@UTEP.For more information, please contact Iweber@utep.edu. 


\title{
Why a Classification Based on Linear Approximation to Dynamical Systems Often Works Well in Nonlinear Cases
}

\author{
Julio Urenda ${ }^{1.2}$ and Vladik Kreinovich ${ }^{2}$ \\ ${ }^{1}$ Department of Mathematical Sciences \\ ${ }^{2}$ Department of Computer Science \\ University of Texas at El Paso \\ $500 \mathrm{~W}$. University \\ El Paso, TX 79968, USA \\ jcurenda@utep.edu, vladik@utep.edu
}

\begin{abstract}
It can be proven that linear dynamical systems exhibit either stable behavior, or unstable behavior, or oscillatory behavior, or transitional behavior. Interesting, the same classification often applies to nonlinear dynamical systems as well. In this paper, we provide a possible explanation for this phenomenon, i.e., we explain why a classification based on linear approximation to dynamical systems often works well in nonlinear cases.
\end{abstract}

\section{Formulation of the Problem}

Dynamical systems are ubiquitous. To describe the state of a real-life system at any given moment of time, we need to know the values $x=\left(x_{1}, \ldots, x_{n}\right)$ of all the quantities that characterize this system. For example, to describe the state of a mechanical system consisting of several pointwise objects, we need to know the position and velocities of all these objects. To describe the state of an electric circuit, we need to know the currents and voltages, etc.

In many real-life situation, the corresponding systems are deterministic - in the sense that the future states of the system are uniquely determined by its current state. Sometimes, to make the system deterministic, we need to enlarge its description so that it incorporates all the objects that affect its dynamics. For example, the system consisting of Earth and Moon is not deterministic in its original form - since the Sun affects its dynamics, but once we add the Sun, we get a system with a deterministic behavior.

The fact that the future dynamics of the system is uniquely determined by its current state means, in particular, that the rate $\dot{x}$ with which the system changes 
is also uniquely determined by its current state, i.e., that we have $\dot{x}=f(x)$, for some function $f(x)$. This equation can be described coordinate-wise, as

$$
\dot{x}_{i}=f_{i}\left(x_{1}, \ldots, x_{n}\right) .
$$

Systems that satisfy such equations are known as dynamical systems; see, e.g., [1].

Simplest case: linear systems. The simplest case is when the rate of change $f_{i}\left(x_{1}, \ldots, x_{n}\right)$ of each variables is a linear function, i.e., when

$$
\dot{x}_{i}=a_{i 0}+\sum_{j=1}^{n} a_{i j} \cdot x_{j} .
$$

In almost all such cases - namely, in all the cases when the matrix $a_{i j}$ is nondegenerate - we can select constants $s_{i}$ so that for the correspondingly shifted variables $y_{i}=x_{i}+s_{i}$, the system gets an even simpler form

$$
\dot{y}_{i}=\sum_{j=1}^{n} a_{i j} \cdot y_{j}
$$

Indeed, substituting $x_{i}=y_{i}-s_{i}$ into the formula (2), and taking into account that $\dot{y}_{i}=\dot{x}_{i}$, we conclude that

$$
\dot{x}_{i}=a_{i 0}+\sum_{j=1}^{n} a_{i j} \cdot\left(y_{j}-s_{j}\right)=a_{i 0}+\sum_{j=1}^{n} a_{i j} \cdot y_{j}-\sum_{j=1}^{n} a_{i j} \cdot s_{j} .
$$

Thus, if we select the value $s_{j}$ for which $a_{i 0}=\sum_{j=1}^{n} a_{i j} \cdot s_{j}$ for each $i$, we will indeed get the formula (2).

For the equation (2), the general solution is well known: it is a linear combination of expressions of the type $t^{k} \cdot \exp (\lambda \cdot t)$, where $\lambda$ is an eigenvalue of the matrix $\left\|a_{i j}\right\|$ - which is, in general, a complex number $\lambda=a+\mathrm{i} \cdot b$, and $k$ is a natural number which does not exceed the multiplicity of this eigenvalue. In real-number terms, we get a linear combination of the expressions $t^{k} \cdot \exp (a \cdot t) \cdot \sin (b \cdot t+\varphi)$.

Depending on the values of $\lambda$, we have the following types of behavior:

- when $a<0$ for all the eigenvalues, then the system is stable: no matter what state we start with, it asymptotically tends to the state $y_{1}=\ldots=$ $y_{n}=0$

- when $a>0$ for at least one eigenvalue, then the system is unstable: the deviation from the 0 state exponentially grows with time;

- when $a=0$ and $b \neq 0$, we get an oscillatory behavior; and

- when $a=b=0$, we get a transitional behavior, when a system linearly (or quadratically etc.) moves from one state to another. 
A similar classification works well in non-linear cases, but why? Interestingly, a similar classification works well for nonlinear dynamical systems as well, but why? In this paper, we will try to explain this fact.

\section{Our Explanation}

We need finite-dimensional approximations. We want to describe how the state $x(t)=\left(x_{1}(t), \ldots, x_{n}(t)\right)$ of a dynamical system changes with time $t$. In general, the set of all possible smooth functions $x_{i}(t)$ is infinite-dimensional, i.e., we need infinitely many parameters to describe it. However, in practice, at any given moment, we can only have finitely many parameters. Thus, it is reasonable to look for finite-parametric approximations. A natural idea is to fix some smooth functions $e_{k}(t)=\left(e_{k 1}(t), \ldots, e_{k n}(t)\right), 1 \leq k \leq K$, and consider linear combinations

$$
x(t)=\sum_{k=1}^{K} c_{k} \cdot e_{k}(t) .
$$

Shift-invariance. For dynamical systems, there is no fixed moment of time. The equations remain the same if we change the starting point for measuring time, i.e., if we replace the original temporal variable $t$ with the new variable $t^{\prime}=t+t_{0}$.

It is therefore reasonable to require that the approximating family (4) be invariant with respect to the same transformation, i.e., in other words, that all shifted functions $e_{k}\left(t+t_{0}\right)$ can also be represented in the same form (4). Let us show that this reasonable requirement explains the above phenomenon.

Comment. This derivation will be similar to the one given in [2].

Towards the explanation. The formula (4) means that for each component $i$, we have

$$
x_{i}(t)=\sum_{k=1}^{K} c_{k} \cdot e_{k i}(t) .
$$

The fact that shifted functions can be represented in this form means that for each $k, i$, and $t_{0}$, we have

$$
e_{k i}\left(t+t_{0}\right)=\sum_{\ell=1}^{K} c_{k \ell i}\left(t_{0}\right) \cdot e_{\ell i}(t),
$$

for some coefficients $c_{k \ell i}\left(t_{0}\right)$ depending on $k, \ell, i$, and $t_{0}$.

Let us fix $i$ and $k$ and select $K$ different moments of time $t_{m}, m=1, \ldots, K$. For these moments of time, (6) takes the form

$$
e_{k i}\left(t_{m}+t_{0}\right)=\sum_{\ell=1}^{K} c_{k \ell i}\left(t_{0}\right) \cdot e_{\ell i}\left(t_{m}\right) .
$$


Thus, we get $K$ linear equations for determining $K$ unknowns $c_{k 1 i}\left(t_{0}\right), \ldots$, $c_{k K i}\left(t_{0}\right)$. Cramer's formula describe the solution to a system of linear equations as a rational (and thus, smooth) function of its coefficients and right-hand sides. Thus, each coefficient $c_{k \ell i}\left(t_{0}\right)$ is a smooth function of the values $e_{k i}\left(t_{m}+t_{0}\right)$ and $e_{\ell i}\left(t_{m}\right)$. Since the functions $e_{k i}(t)$ are smooth, the dependence of the coefficients $c_{k \ell i}\left(t_{0}\right)$ on $t_{0}$ is also differentiable.

Since all the functions involved in the formula (6) are differentiable, we can differentiate this formula with respect to $t_{0}$ and get

$$
\dot{e}_{k i}\left(t+t_{0}\right)=\sum_{\ell=1}^{K} \dot{c}_{k \ell i}\left(t_{0}\right) \cdot e_{\ell i}(t) .
$$

In particular, for $t_{0}=0$, we get

$$
\dot{e}_{k i}(t)=\sum_{\ell=1}^{K} a_{k \ell i} \cdot e_{\ell i}(t),
$$

where we denoted $a_{k \ell i} \stackrel{\text { def }}{=} \dot{c}_{k \ell i}\left(t_{0}\right)$.

So, we conclude that the functions $e_{k i}(t)$ satisfy the system of linear differential equations with constant coefficients - and we have already mentioned that the solutions to such systems are exactly the functions leading to a known classification of linear dynamical system behaviors.

This explains why for nonlinear systems, we also naturally observe similar types of behavior.

\section{Acknowledgments}

This work was supported in part by the US National Science Foundation grants 1623190 (A Model of Change for Preparing a New Generation for Professional Practice in Computer Science) and HRD-1242122 (Cyber-ShARE Center of Excellence).

\section{References}

[1] M. W. Hirsch, S. Smale, and R. L. Devaney, Differential Equations, Dynamical Systems, and an Introduction to Chaos, Academic Press, Waltham, Massachisetts, 2013.

[2] H. T. Nguyen and V. Kreinovich, Applications of Continuous Mathematics to Computer Science, Kluwer, Dordrecht, 1997. 